\title{
Painlevé IV, $\sigma$-Form and the Deformed Hermite Unitary Ensembles
}

\author{
Mengkun Zhu*a,b, Dan Wang ${ }^{\dagger b}$, and Yang Chen ${ }^{\ddagger b}$ \\ ${ }^{\mathrm{a}}$ School of Mathematics and Statistics, Qilu University of Technology (Shandong \\ Academy of Sciences), Jinan 250353, China \\ ${ }^{\mathrm{b}}$ Department of Mathematics, Faculty of Science and Technology, University of Macau, \\ Avenida da Universidade, Taipa, Macau, China
}

\begin{abstract}
We study the Hankel determinant generated by a deformed Hermite weight with one jump $w(z, t, \gamma)=e^{-z^{2}+t z}|z-t|^{\gamma}(A+B \theta(z-t))$, where $A \geq 0$, $A+B \geq 0, t \in \mathbf{R}, \gamma>-1$ and $z \in \mathbf{R}$. By using the ladder operators for the corresponding monic orthogonal polynomials, and their relative compatibility conditions, we obtain a series of difference and differential equations to describe the relations among $\alpha_{n}, \beta_{n}, R_{n}(t)$ and $r_{n}(t)$. Especially, we find that the auxiliary quantities $R_{n}(t)$ and $r_{n}(t)$ satisfy the coupled Riccati equations, and $R_{n}(t)$ satisfies a particular Painlevé IV equation. Based on above results, we show that $\sigma_{n}(t)$ and $\hat{\sigma}_{n}(t)$, two quantities related to the Hankel determinant and $R_{n}(t)$, satisfy the continuous and discrete $\sigma-$ form equations, respectively. In the end, we also discuss the large $n$ asymptotic behavior of $R_{n}(t)$, which produce the expansion of the logarithmic of the Hankel determinant and the asymptotic of the second order differential equation of the monic orthogonal polynomials.

Keywords: Ladder operators, Hermite unitary ensembles, $\sigma$-form, Painlevé IV.

MSC: 15B52, 42C05, 33E17
\end{abstract}

\section{Introduction}

The joint probability density function of the eigenvalues $\left\{z_{i}\right\}_{i=1}^{n}$,

$$
p\left(z_{1}, z_{2}, \cdots, z_{n}\right)=\frac{1}{n ! D_{n}\left(w_{0}\right)} \prod_{1 \leq i<k \leq n}\left(z_{k}-z_{i}\right)^{2} \prod_{j=1}^{n} w_{0}\left(z_{j}\right),
$$

*Zhu_mengkun@163.com

†Corresponding author: bohewan@126.com

‡yangbrookchen@yahoo.co.uk 
is a well known fact in the theory of random matrix ensembles [6 8, 13], where $w_{0}(z)$ is a weight function on the interval $(a, b)$ and has the finite moments, i. e.

$$
\mu_{k}:=\int_{a}^{b} z^{k} w_{0}(z) d z, \quad k \in\{0,1,2, \cdots\} .
$$

Here $D_{n}\left(w_{0}\right)$ is the normalization constant

$$
D_{n}\left(w_{0}\right)=\frac{1}{n !} \int_{[a, b]^{n}} \prod_{1 \leq i<k \leq n}\left(z_{k}-z_{i}\right)^{2} \prod_{j=1}^{n} w_{0}\left(z_{j}\right) d z_{j},
$$

so that

$$
\int_{[a, b]^{n}} p\left(z_{1}, z_{2}, \cdots, z_{n}\right) d z_{1} d z_{2} \cdots d z_{n}=1 .
$$

In this paper, we consider

$$
w(z, t, \gamma)=e^{-z^{2}+t z}|z-t|^{\gamma}(A+B \theta(z-t)),
$$

where $A \geq 0, A+B \geq 0, t \in \mathbf{R}, \gamma>-1$ and $z \in \mathbf{R}$, which

$$
w_{0}(z)=e^{-z^{2}+t z}, \quad t \in \mathbf{R}, \quad z \in \mathbf{R},
$$

corresponds to the deformed Hermite (or Gaussian) unitary ensemble. Here $\theta(x)$ is the Heaviside function, i.e.

$$
\theta(x)=\left\{\begin{array}{ll}
1, & x>0 \\
0, & x \leq 0
\end{array} .\right.
$$

According to the general theory of orthogonal polynomials of one variable, the normalization constant (1.1) has the two more alternative representation

$$
D_{n}(w)=\operatorname{det}\left(\mu_{i+j}(t)\right)_{i, j=0}^{n-1}=\operatorname{det}\left(\int_{\mathbf{R}} z^{i+j} w(z, t) d z\right)_{i, j=0}^{n-1}=\prod_{k=0}^{n-1} h_{k}(t),
$$

where the determinant of the moment matrix $\mu_{i+j}(t)$ is the Hankel determinant, and $\left\{h_{k}(t)\right\}_{k=0}^{n}$ is the square of the $L^{2}$ norm of the sequence of polynomials $\left\{P_{k}(z)\right\}_{k=0}^{n}$.

Min and Chen [14] have studied the Painlevé transcendents and the Hankel determinants generated by a discontinuous Gaussian weight

$$
w\left(x, t_{1}, t_{2}\right)=e^{-x^{2}}\left(A+B_{1} \theta\left(x-t_{1}\right)+B_{2} \theta\left(x-t_{2}\right)\right),
$$

where $A_{1}, B_{1}$ and $B_{2}$ are constants, $A \geq 0, A+B_{1} \geq 0, A+B_{1}+B_{2} \geq 0, t_{1}<$ $t_{2}, x \in \mathbf{R}$. They considered the Gaussian weight with a single jump $\left(B_{1}\right.$ or $B_{2}$ $=0)$ or two jumps $\left(B_{1} \neq 0\right.$ and $\left.B_{2} \neq 0\right)$, and proved their auxiliary quantities satisfy the second order difference and differential equations, respectively, via the ladder operators and supplementary conditions.

Let $P_{n}(z, t, \gamma)$ be the monic polynomials of degree $n$ orthogonal with respect to (1.2),

$$
\int_{\mathbf{R}} P_{j}(z, t, \gamma) P_{i}(z, t, \gamma) w(z, t, \gamma) d z=h_{j}(t, \gamma) \delta_{j i},
$$


where $j, i \in\{0,1,2, \cdots\}$, and $\delta_{j i}$ denotes the Kronecker delta. It follows from the orthogonality relations that

$$
z P_{n}(z, t, \gamma)=P_{n+1}(z, t, \gamma)+\alpha_{n} P_{n}(z, t, \gamma)+\beta_{n} P_{n-1}(z, t, \gamma), \quad n \geq 0
$$

together with the initial conditions

$$
P_{0}(z, t, \gamma):=1, \quad \beta_{0} P_{-1}(z, t, \gamma):=0 .
$$

The monic polynomials $P_{n}(z, t)$ have the monomial expansion

$$
P_{n}(z, t, \gamma)=z^{n}+\mathbf{p}(n, t, \gamma) z^{n-1}+\cdots+P_{n}(0, t, \gamma),
$$

and we will see that the coefficient of $z^{n-1}, \mathbf{p}(n, t, \gamma)$, play a significant role in the following discussions. Note that due to the $t$ dependence of the weight, the coefficients of the polynomials and the recurrence coefficients $\alpha_{n}$ and $\beta_{n}$ also depend on $t$, the position of the jump.

From (1.5) and (1.6), for $n \in\{0,1,2, \cdots\}$,

$$
\alpha_{n}=\mathbf{p}(n, t, \gamma)-\mathbf{p}(n+1, t, \gamma)
$$

and

$$
\beta_{n}=\frac{h_{n}(t, \gamma)}{h_{n-1}(t, \gamma)}
$$

A telescopic sum of (1.7), it yields

$$
\sum_{j=0}^{n-1} \alpha_{j}=-\mathbf{p}(n, t, \gamma)
$$

The paper is organized up as follow. In section 2, we derive the ladder operators and the compatibility conditions $\left(S_{1}\right),\left(S_{2}\right)$ and $\left(S_{2}^{\prime}\right)$ with respect to the weight (1.2). In section 3 , we obtain some important identities on the auxiliary quantities $R_{n}(t)$ and $r_{n}(t)$ via applying the ladder operators. In section 4 , we are interested in the parameter of the weight (1.2), and we show that $r_{n}(t)$ and $R_{n}(t)$ satisfy the coupled Riccati equations. Section 5 is devoted to discuss two quantities $\sigma_{n}(t)$ and $\hat{\sigma}_{n}(t)$, which are allied to the Hankel determinant and $R_{n}(t)$. We prove that they satisfy the continuous or discrete $\sigma$-form equations, respectively. The final section, we make use of the equations in section $2-5$ to show that $R_{n}(t)$ satisfy a particular Painlevé IV equation. Based on this equation, we obtain the large $n$ asymptotic of the auxiliary quantity $R_{n}(t)$. Moreover, the logarithmic of the Hankel determinant $D_{n}(t)$ and the asymptotic of the second order differential equation of $P_{n}(z, t, \gamma)$ are also obtained.

\section{Ladder operators and compatibility con- ditions}

A detailed proof of the lowering ladder operator, which is included in the ladder operators [1], is shown in the following theorem. For convenience, we 
denote

$$
\begin{aligned}
w(z, t, \gamma) & =e^{-z^{2}+t z}|z-t|^{\gamma}(A+B \theta(z-t)), \\
w_{0}(z, t) & =e^{-z^{2}+t z} \\
w_{J}(z, t) & =A+B \theta(z-t),
\end{aligned}
$$

where $A \geq 0, A+B \geq 0, t \in \mathbf{R}, \gamma>-1$ and $z \in \mathbf{R}$. Meanwhile, we do not always write down the $t$ or $\gamma$ dependence in $P_{n}(z, t, \gamma), w(z, t, \gamma), w_{0}(z, t)$, $w_{J}(z, t), h_{n}(t, \gamma), \alpha_{n}(t, \gamma)$ and $\beta_{n}(t, \gamma)$ unless it is needed.

Theorem 2.1. The monic orthogonal polynomials with respect to the weight (1.2) satisfy the following differentiation formula:

$$
P_{n}^{\prime}(z)=\beta_{n} A_{n}(z) P_{n-1}(z)-B_{n}(z) P_{n}(z),
$$

where

$$
\begin{gathered}
A_{n}(z)=\frac{1}{h_{n}} \int_{\boldsymbol{R}} P_{n}^{2}(y) \frac{v_{0}^{\prime}(z)-v_{0}^{\prime}(y)}{z-y} w(y) d y+a_{n}(t), \\
B_{n}(z)=\frac{1}{h_{n-1}} \int_{\boldsymbol{R}} P_{n}(y) P_{n-1}(y) \frac{v_{0}^{\prime}(z)-v_{0}^{\prime}(y)}{z-y} w(y) d y+b_{n}(t), \\
a_{n}(t)=\frac{\gamma}{h_{n}} \int_{\boldsymbol{R}} \frac{P_{n}^{2}(y)}{(z-y)(y-t)} w(y) d y
\end{gathered}
$$

and

$$
b_{n}(t)=\frac{\gamma}{h_{n-1}} \int_{\boldsymbol{R}} \frac{P_{n}(y) P_{n-1}(y)}{(z-y)(y-t)} w(y) d y .
$$

Here

$$
v_{0}(z)=-\ln w_{0}(z)
$$

Proof. Firstly, we note [4]

$$
\frac{\partial\left(|z-t|^{\gamma}\right)}{\partial z}=\left((z-t)^{\gamma}-(t-z)^{\gamma}\right) \delta(z-t)+\frac{\gamma|z-t|^{\gamma}}{z-t}
$$

which is obtained by writing

$$
|z-t|^{\gamma}=(z-t)^{\gamma} \theta(z-t)+(t-z)^{\gamma} \theta(t-z) .
$$

From

$$
P_{n}^{\prime}(z)=\sum_{k=0}^{n-1} C_{n, k} P_{k}(z),
$$

where $C_{n, k}$ is determined from the orthogonality relations

$$
C_{n, k}=\frac{1}{h_{k}} \int_{\mathbf{R}} P_{n}^{\prime}(y) P_{k}(y) \omega(y) d y .
$$


Using the integration by parts and noting the weight (1.2) vanishes at the endpoints of the orthogonality interval, we find

$$
\begin{aligned}
C_{n, k} & =\frac{1}{h_{k}} \int_{\mathbf{R}} P_{n}^{\prime}(y) P_{k}(y) w(y) d y=-\frac{1}{h_{k}} \int_{\mathbf{R}} P_{n}(y) P_{k}(y) w^{\prime}(y) d y \\
& =-\frac{1}{h_{k}} \int_{\mathbf{R}} P_{n}(y) P_{k}(y)\left(v_{0}^{\prime}(z)-v_{0}^{\prime}(y)\right) w(y) d y-\frac{\gamma}{h_{k}} \int_{\mathbf{R}} P_{n}(y) P_{k}(y) \frac{w(y)}{y-t} d y
\end{aligned}
$$

straightforward,

$$
\begin{aligned}
& P_{n}^{\prime}(z) \\
= & \sum_{k=0}^{n-1}\left(-\frac{1}{h_{k}} \int_{\mathbf{R}} P_{n}(y) P_{k}(y)\left(v_{0}^{\prime}(z)-v_{0}^{\prime}(y)\right) w(y) d y-\frac{\gamma}{h_{k}} \int_{\mathbf{R}} P_{n}(y) P_{k}(y) \frac{w(y)}{y-t} d y\right) P_{k}(z) \\
= & -\int_{\mathbf{R}} P_{n}(y) \sum_{k=0}^{n-1} \frac{P_{k}(y) P_{k}(z)}{h_{k}}\left(v_{0}^{\prime}(z)-v_{0}^{\prime}(y)\right) w(y) d y-\gamma \int_{\mathbf{R}} P_{n}(y) \sum_{k=0}^{n-1} \frac{P_{k}(y) P_{k}(z)}{h_{k}} \frac{w(y)}{y-t} d y \\
= & -\frac{1}{h_{n-1}} \int_{\mathbf{R}} \frac{v_{0}^{\prime}(z)-v_{0}^{\prime}(y)}{z-y} P_{n}(y) P_{n-1}(y) w(y) d y P_{n}(z) \\
& +\frac{1}{h_{n-1}} \int_{\mathbf{R}} \frac{v_{0}^{\prime}(z)-v_{0}^{\prime}(y)}{z-y} P_{n}^{2}(y) w(y) d y P_{n-1}(z) \\
& -\frac{\gamma}{h_{n-1}} \int_{\mathbf{R}} P_{n}(y) P_{n-1}(y) \frac{w(y)}{(z-y)(y-t)} d y P_{n}(z) \\
& +\frac{\gamma}{h_{n-1}} \int_{\mathbf{R}} P_{n}^{2}(y) \frac{w(y)}{(z-y)(y-t)} d y P_{n-1}(z),
\end{aligned}
$$

where we have used the Christoffel-Darboux formula [17]. Applying (1.8), we arrive the desired result.

Corollary 2.2. Two identities

$$
\gamma \int_{\boldsymbol{R}} \frac{P_{n}^{2}(y) w(y)}{y-t} d y=\int_{\boldsymbol{R}} P_{n}^{2}(y) v_{0}^{\prime}(y) w(y) d y
$$

and

$$
\frac{\gamma}{h_{n-1}} \int_{\boldsymbol{R}} P_{n}(y) P_{n-1}(y) \frac{w(y)}{y-t} d y=\frac{1}{h_{n-1}} \int_{\boldsymbol{R}} P_{n}(y) P_{n-1}(y) v_{0}^{\prime}(y) w(y) d y-n
$$

Proof. From the proof of theorem 2.1, we see that

$$
\frac{1}{h_{n}} \int_{\mathbf{R}} P_{n}^{\prime}(y) P_{n}(y) w(y) d y=0 .
$$

Applying integration by parts into the left side of above equation,

$\frac{1}{h_{n}} \int_{\mathbf{R}} P_{n}^{\prime}(y) P_{n}(y) w(y) d y=\frac{1}{h_{n}} \int_{\mathbf{R}} P_{n}^{2}(y) v_{0}^{\prime}(y) w(y) d y-\frac{\gamma}{h_{n}} \int_{\mathbf{R}} P_{n}^{2}(y) \frac{w(y)}{y-t} d y$, which gives (2.8).

The same steps to do below equation,

$$
\frac{1}{h_{n-1}} \int_{\mathbf{R}} P_{n}^{\prime}(y) P_{n-1}(y) w(y) d y=n,
$$

we obtain (2.9). 
Remark 1. The equation (2.1) is called a lowering operator, see [2, 3, 9, 14, [22-24]. With the help of (2.1) and (2.10), $P_{n}(z)$ also satisfies the raising operator equation

$$
P_{n-1}^{\prime}(z)=-A_{n-1}(z) P_{n}(z)+\left(B_{n}(z)+v_{0}^{\prime}(z)\right) P_{n-1}(z) .
$$

Note that $A_{n}(z)$ and $B_{n}(z)$ are not independent, a direct calculation produces two fundamental supplementary conditions [2] valid for all $z$.

Theorem 2.3. The functions $A_{n}(z)$ and $B_{n}(z)$ satisfy the conditions:

$$
\begin{gathered}
B_{n}(z)+B_{n+1}(z)=\left(z-\alpha_{n}\right) A_{n}(z)-v_{0}^{\prime}(z) \\
1+\left(z-\alpha_{n}\right)\left(B_{n+1}(z)-B_{n}(z)\right)=\beta_{n+1} A_{n+1}(z)-\beta_{n} A_{n-1}(z) .
\end{gathered}
$$

Proof. Using (1.8), the recurrence relation $\left(z-\alpha_{n}\right) P_{n}(z, t, \gamma)=P_{n+1}(z, t, \gamma)+$ $\beta_{n} P_{n-1}(z, t, \gamma)$ has a restatement, i. e.

$$
\frac{\left(z-\alpha_{n}\right) P_{n}(z, t, \gamma)}{h_{n}}=\frac{P_{n+1}(z, t, \gamma)}{h_{n}}+\frac{P_{n-1}(z, t, \gamma)}{h_{n-1}} .
$$

From the definitions of $A_{n}(z)$ and $B_{n}(z)$, (2.2) and (2.3), and substituting (2.12) into below computation, we have

$$
\begin{aligned}
& B_{n}(z)+B_{n+1}(z) \\
= & \int_{\mathbf{R}} P_{n}(y)\left(\frac{P_{n+1}(y)}{h_{n}}+\frac{P_{n-1}(y)}{h_{n-1}}\right) \frac{v_{0}^{\prime}(z)-v_{0}^{\prime}(y)}{z-y} w(y) d y \\
& +\gamma \int_{\mathbf{R}} P_{n}(y)\left(\frac{P_{n+1}(y)}{h_{n}}+\frac{P_{n-1}(y)}{h_{n-1}}\right) \frac{w(y)}{(z-y)(y-t)} d y \\
& =\frac{1}{h_{n}} \int_{\mathbf{R}}\left(y-\alpha_{n}\right) P_{n}^{2}(y) \frac{v_{0}^{\prime}(z)-v_{0}^{\prime}(y)}{z-y} w(y) d y+\frac{\gamma}{h_{n}} \int_{\mathbf{R}} \frac{\left(y-\alpha_{n}\right) P_{n}^{2}(y) w(y)}{(z-y)(y-t)} d y \\
& =-\frac{1}{h_{n}} \int_{\mathbf{R}} P_{n}^{2}(y)\left(v_{0}^{\prime}(z)-v_{0}^{\prime}(y)\right) w(y) d y-\frac{\gamma}{h_{n}} \int_{\mathbf{R}} \frac{P_{n}^{2}(y) w(y)}{y-t} d y \\
& +\frac{1}{h_{n}} \int_{\mathbf{R}}\left(z-\alpha_{n}\right) P_{n}^{2}(y) \frac{v_{0}^{\prime}(z)-v_{0}^{\prime}(y)}{z-y} w(y) d y+\frac{\gamma}{h_{n}} \int_{\mathbf{R}} \frac{\left(z-\alpha_{n}\right) P_{n}^{2}(y) w(y)}{(z-y)(y-t)} d y \\
& =\left(z-\alpha_{n}\right) A_{n}(z)-v_{0}^{\prime}(z)+\frac{1}{h_{n}} \int_{\mathbf{R}} P_{n}^{2}(y) v_{0}^{\prime}(y) w(y) d y-\frac{\gamma}{h_{n}} \int_{\mathbf{R}} \frac{P_{n}^{2}(y) w(y)}{y-t} d y,
\end{aligned}
$$

where

$$
y-\alpha_{n}=(y-z)+\left(z-\alpha_{n}\right)
$$

and (2.8) are used.The proof of (2.10) is completed. 
Similarly steps, by using the definition of $A_{n}(z)$ and (1.8), we have

$$
\begin{aligned}
& \beta_{n+1} A_{n+1}(z)-\beta_{n} A_{n-1}(z) \\
= & \frac{1}{h_{n}} \int_{\mathbf{R}} \frac{v_{0}^{\prime}(z)-v_{0}^{\prime}(y)}{z-y} P_{n+1}^{2}(y) w(y) d y+\frac{\gamma}{h_{n}} \int_{\mathbf{R}} \frac{P_{n+1}^{2}(y)}{(z-y)(y-t)} w(y) d y \\
& -\frac{\beta_{n}}{h_{n-1}} \int_{\mathbf{R}} \frac{v_{0}^{\prime}(z)-v_{0}^{\prime}(y)}{z-y} P_{n-1}^{2}(y) w(y) d y-\frac{\gamma \beta_{n}}{h_{n-1}} \int_{\mathbf{R}} \frac{P_{n-1}^{2}(y) w(y)}{(z-y)(y-t)} d y \\
= & \int_{\mathbf{R}} \frac{v_{0}^{\prime}(z)-v_{0}^{\prime}(y)}{z-y}\left(\frac{P_{n+1}^{2}(y)}{h_{n}}-\frac{\beta_{n} P_{n-1}^{2}(y)}{h_{n-1}}\right) w(y) d y \\
& +\gamma \int_{\mathbf{R}}\left(\frac{P_{n+1}^{2}(y)}{h_{n}}-\frac{\beta_{n} P_{n-1}^{2}(y)}{h_{n-1}}\right) \frac{w(y)}{(z-y)(y-t)} d y .
\end{aligned}
$$

On the other hand, Applying (2.9) and (2.12), we consider

$$
\begin{aligned}
& \left(z-\alpha_{n}\right)\left(B_{n+1}(z)-B_{n}(z)\right) \\
& =\left(z-\alpha_{n}\right)\left(\int_{\mathbf{R}} \frac{v_{0}^{\prime}(z)-v_{0}^{\prime}(y)}{z-y}\left(\frac{P_{n+1}(y)}{h_{n}}-\frac{P_{n-1}(y)}{h_{n-1}}\right) P_{n}(y) w(y) d y\right. \\
& +\gamma \int_{0}^{\infty} \frac{P_{n}(y)}{(z-y)(y-t)}\left(\frac{P_{n+1}(y)}{h_{n}}-\frac{P_{n-1}(y)}{h_{n-1}}\right) w(y) d y \\
& =\int_{\mathbf{R}}\left(v_{0}^{\prime}(z)-v_{0}^{\prime}(y)\right)\left(\frac{P_{n+1}(y)}{h_{n}}-\frac{P_{n-1}(y)}{h_{n-1}}\right) P_{n}(y) w(y) d y \\
& +\int_{\mathbf{R}} \frac{v_{0}^{\prime}(z)-v_{0}^{\prime}(y)}{z-y}\left(\frac{P_{n+1}^{2}(y)}{h_{n}^{2}}-\frac{P_{n-1}^{2}(y)}{h_{n-1}^{2}}\right) h_{n} w(y) d y \\
& +\gamma \int_{\mathbf{R}} \frac{P_{n}(y)}{y-t}\left(\frac{P_{n+1}(y)}{h_{n}}-\frac{P_{n-1}(y)}{h_{n-1}}\right) w(y) d y \\
& +\gamma \int_{\mathbf{R}} \frac{w(y)}{(z-y)(y-t)}\left(\frac{P_{n+1}^{2}(y)}{h_{n}^{2}}-\frac{P_{n-1}^{2}(y)}{h_{n-1}^{2}}\right) h_{n} d y \\
& =-\left(\frac{1}{h_{n}} \int_{\mathbf{R}} P_{n+1}(y) P_{n}(y) v_{0}^{\prime}(y) w(y) d y-\frac{\gamma}{h_{n}} \int_{\mathbf{R}} \frac{P_{n}(y) P_{n+1}(y) w(y)}{y-t} d y\right) \\
& +\left(\frac{1}{h_{n-1}} \int_{\mathbf{R}} P_{n}(y) P_{n-1}(y) v_{0}^{\prime}(y) w(y) d y-\frac{\gamma}{h_{n-1}} \int_{\mathbf{R}} \frac{P_{n-1}(y) P_{n}(y) w(y)}{y-t} d y\right) \\
& +\int_{\mathbf{R}} \frac{v_{0}^{\prime}(z)-v_{0}^{\prime}(y)}{z-y}\left(\frac{P_{n+1}^{2}(y)}{h_{n}}-\frac{\beta_{n} P_{n-1}^{2}(y)}{h_{n-1}}\right) w(y) d y \\
& +\gamma \int_{\mathbf{R}} \frac{w(y)}{(z-y)(y-t)}\left(\frac{P_{n+1}^{2}(y)}{h_{n}}-\frac{\beta_{n} P_{n-1}^{2}(y)}{h_{n-1}}\right) d y \\
& =\int_{\mathbf{R}} \frac{v_{0}^{\prime}(z)-v_{0}^{\prime}(y)}{z-y}\left(\frac{P_{n+1}^{2}(y)}{h_{n}}-\frac{\beta_{n} P_{n-1}^{2}(y)}{h_{n-1}}\right) w(y) d y \\
& +\gamma \int_{\mathbf{R}} \frac{w(y)}{(z-y)(y-t)}\left(\frac{P_{n+1}^{2}(y)}{h_{n}}-\frac{\beta_{n} P_{n-1}^{2}(y)}{h_{n-1}}\right) d y-1 .
\end{aligned}
$$

(2.14) minus (2.15) follows

$$
\beta_{n+1} A_{n+1}(z)-\beta_{n} A_{n-1}(z)-\left(z-\alpha_{n}\right)\left(B_{n+1}(z)-B_{n}(z)\right)=1,
$$

and we arrive (2.11). 
Remark 2. 2.10) and (2.11) are the well-known supplementary conditions $\left(S_{1}\right)$ and $\left(S_{2}\right)$, respectively. $\left(S_{1}\right)$ and $\left(S_{2}\right)$ have been applied to random matrix theory in [20].

We produces an identity involving $\sum_{k=0}^{n-1} A_{k}(z)$ by the combination of $\left(S_{1}\right)$ and $\left(S_{2}\right)$.

Theorem 2.4. $A_{n}(z), B_{n}(z)$ and $\sum_{k=0}^{n-1} A_{k}(z)$ satisfy the identity

$$
B_{n}^{2}(z)+v_{0}^{\prime}(z) B_{n}(z)+\sum_{k=0}^{n-1} A_{k}(z)=\beta_{n} A_{n}(z) A_{n-1}(z) .
$$

Proof. Using $\left(S_{1}\right)$ with multiplying $\left(S_{2}\right)$ by $A_{n}(z)$ on both sides, we have

$$
\begin{aligned}
& A_{n}(z)+B_{n+1}^{2}(z)-B_{n}^{2}(z)+v_{0}^{\prime}(z)\left(B_{n+1}(z)-B_{n}(z)\right) \\
= & \beta_{n+1} A_{n+1}(z) A_{n}(z)-\beta_{n} A_{n}(z) A_{n-1}(z) .
\end{aligned}
$$

Taking a telescopic sum together with the appropriate "initial conditions", $B_{0}(z)=0$ and $\beta_{0} A_{-1}(z)=0$, we obtain the desired result.

Remark 3. The compatibility conditions $\left(S_{1}\right),\left(S_{2}\right)$ and $\left(S_{2}^{\prime}\right)$ are valid for $z \in C \cup\{\infty\}$.

Using Theorem 2.1 and Theorem 2.3, we obtain the second order linear ordinary differential equation satisfied by $P_{n}(z)$.

Theorem 2.5. The monic orthogonal polynomials $P_{n}(z)$ satisfy the second order differential equation,

$$
P_{n}^{\prime \prime}(z)+Q_{n}(z) P_{n}^{\prime}(z)+T_{n}(z) P_{n}(z)=0
$$

where

$$
\begin{gathered}
Q_{n}(z)=-v_{0}^{\prime}(z)-\frac{A_{n}^{\prime}(z)}{A_{n}(z)} \\
T_{n}(z)=B_{n}^{\prime}(z)-\frac{B_{n}(z) A_{n}^{\prime}(z)}{A_{n}(z)}+\beta_{n} A_{n}(z) A_{n-1}(z)-B_{n}^{2}(z)-B_{n}(z) v_{0}^{\prime}(z) .
\end{gathered}
$$

Proof. See [5, 14, 21, 24].

\section{Deformed Hermite weight}

Recalling Theorem 2.1,

$$
\begin{gathered}
A_{n}(z)=\frac{1}{h_{n}} \int_{\mathbf{R}} P_{n}^{2}(y) \frac{v_{0}^{\prime}(z)-v_{0}^{\prime}(y)}{z-y} w(y) d y+a_{n}(t), \\
B_{n}(z)=\frac{1}{h_{n-1}} \int_{\mathbf{R}} P_{n}(y) P_{n-1}(y) \frac{v_{0}^{\prime}(z)-v_{0}^{\prime}(y)}{z-y} w(y) d y+b_{n}(t),
\end{gathered}
$$




$$
a_{n}(t)=\frac{\gamma}{h_{n}} \int_{\mathbf{R}} \frac{P_{n}^{2}(y)}{(z-y)(y-t)} w(y) d y
$$

and

$$
b_{n}(t)=\frac{\gamma}{h_{n-1}} \int_{\mathbf{R}} \frac{P_{n}(y) P_{n-1}(y)}{(z-y)(y-t)} w(y) d y,
$$

we have the following expression of $A_{n}(z)$ and $B_{n}(z)$.

Theorem 3.1. As $n \rightarrow \infty$, it yields

$$
A_{n}(z)=2+\frac{R_{n}(t)}{z}+\frac{\gamma+t R_{n}(t)}{z^{2}}+\frac{\gamma \alpha_{n}+\gamma t+t^{2} R_{n}(t)}{z^{3}}+\mathcal{O}\left(z^{-4}\right)
$$

and

$$
B_{n}(z)=\frac{r_{n}(t)}{z}+\frac{t r_{n}(t)}{z^{2}}+\frac{\gamma \beta_{n}+t^{2} r_{n}(t)}{z^{3}}+\mathcal{O}\left(z^{-4}\right),
$$

where

$$
R_{n}(t)=\frac{\gamma}{h_{n}} \int_{\mathbf{R}} \frac{P_{n}^{2}(y) w(y)}{y-t} d y
$$

and

$$
r_{n}(t)=\frac{\gamma}{h_{n-1}} \int_{\mathbf{R}} \frac{P_{n}(y) P_{n-1}(y) w(y)}{y-t} d y .
$$

Proof. As $n \rightarrow \infty$,

$$
\begin{aligned}
\frac{1}{z-y} & =\frac{1}{z} \cdot \frac{1}{1-\frac{y}{z}}=\frac{1}{z}\left(1+\frac{y}{z}+\frac{y^{2}}{z^{2}}+\mathcal{O}\left(z^{-3}\right)\right) \\
& =\frac{1}{z}+\frac{y}{z^{2}}+\frac{y^{2}}{z^{3}}+\mathcal{O}\left(z^{-4}\right)
\end{aligned}
$$

we can rewrite $a_{n}(t)$ and $b_{n}(t)$, respectively,

$$
\begin{aligned}
a_{n}(t)= & \frac{\gamma}{z h_{n}} \int_{\mathbf{R}} \frac{P_{n}^{2}(y) w(y)}{y-t} d y+\frac{\gamma}{z^{2} h_{n}} \int_{\mathbf{R}} \frac{y P_{n}^{2}(y) w(y)}{y-t} d y+\frac{\gamma}{z^{3} h_{n}} \int_{\mathbf{R}} \frac{y^{2} P_{n}^{2}(y) w(y)}{y-t} d y \\
& +\mathcal{O}\left(z^{-4}\right) \\
b_{n}(t)= & \frac{\gamma}{z h_{n-1}} \int_{\mathbf{R}} \frac{P_{n}(y) P_{n-1}(y) w(y)}{y-t} d y+\frac{\gamma}{z^{2} h_{n-1}} \int_{\mathbf{R}} \frac{y P_{n}(y) P_{n-1}(y) w(y)}{y-t} d y \\
& +\frac{\gamma}{z^{3} h_{n-1}} \int_{\mathbf{R}} \frac{y^{2} P_{n}(y) P_{n-1}(y) w(y)}{y-t} d y+\mathcal{O}\left(z^{-4}\right) .
\end{aligned}
$$

Note

$$
\begin{aligned}
& \frac{\gamma}{h_{n}} \int_{\mathbf{R}} \frac{y P_{n}^{2}(y) w(y)}{y-t} d y=\frac{\gamma}{h_{n}} \int_{\mathbf{R}} \frac{((y-t)+t) P_{n}^{2}(y) w(y)}{y-t} d y \\
= & \frac{\gamma}{h_{n}}\left(\int_{\mathbf{R}} P_{n}^{2}(y) w(y) d y+t \int_{\mathbf{R}} \frac{P_{n}^{2}(y) w(y)}{y-t} d y\right) \\
= & \gamma+\frac{\gamma t}{h_{n}} \int_{\mathbf{R}} \frac{P_{n}^{2}(y) w(y)}{y-t} d y,
\end{aligned}
$$




$$
\begin{aligned}
& \frac{\gamma}{h_{n}} \int_{\mathbf{R}} \frac{y^{2} P_{n}^{2}(y) w(y)}{y-t} d y=\frac{\gamma}{h_{n}} \int_{\mathbf{R}} \frac{\left(\left(y^{2}-t^{2}\right)+t^{2}\right) P_{n}^{2}(y) w(y)}{y-t} d y \\
= & \frac{\gamma}{h_{n}}\left(\int_{\mathbf{R}}(y+t) P_{n}^{2}(y) w(y) d y+t^{2} \int_{\mathbf{R}} \frac{P_{n}^{2}(y) w(y)}{y-t} d y\right) \\
= & \frac{\gamma}{h_{n}}\left(\alpha_{n} h_{n}+t h_{n}+t^{2} \int_{\mathbf{R}} \frac{P_{n}^{2}(y) w(y)}{y-t} d y\right) \\
= & \gamma \alpha_{n}+\gamma t+\frac{\gamma t^{2}}{h_{n}} \int_{\mathbf{R}} \frac{P_{n}^{2}(y) w(y)}{y-t} d y, \quad(3.8) \\
& \frac{\gamma}{h_{n-1}} \int_{\mathbf{R}} \frac{y P_{n}(y) P_{n-1}(y) w(y)}{y-t} d y=\frac{\gamma}{h_{n-1}} \int_{\mathbf{R}} \frac{((y-t)+t) P_{n}(y) P_{n-1}(y) w(y)}{y-t} d y \\
= & \frac{\gamma}{h_{n-1}}\left(\int_{\mathbf{R}} P_{n}(y) P_{n-1}(y) w(y) d y+t \int_{\mathbf{R}} \frac{P_{n}(y) P_{n-1}(y) w(y)}{y-t} d y\right) \\
= & \frac{\gamma t}{h_{n-1}} \int_{\mathbf{R}} \frac{P_{n}(y) P_{n-1}(y) w(y)}{y-t} d y, \\
\text { and } & \frac{\gamma}{h_{n-1}} \int_{\mathbf{R}} \frac{y^{2} P_{n}(y) P_{n-1}(y) w(y)}{y-t} d y=\frac{\gamma}{h_{n-1}} \int_{\mathbf{R}} \frac{\left(\left(y^{2}-t^{2}\right)+t^{2}\right) P_{n}(y) P_{n-1}(y) w(y)}{y-t} d y \\
= & \frac{\gamma}{h_{n-1}}\left(\int_{\mathbf{R}}(y+t) P_{n}(y) P_{n-1}(y) w(y) d y+t^{2} \int_{\mathbf{R}} \frac{P_{n}(y) P_{n-1}(y) w(y)}{y-t} d y\right) \\
= & \frac{\gamma}{h_{n-1}}\left(\beta_{n} h_{n-1}+t^{2} \int_{\mathbf{R}} \frac{P_{n}(y) P_{n-1}(y) w(y)}{y-t} d y\right) \\
= & \gamma \beta_{n}+\frac{\gamma t^{2}}{h_{n-1}} \int_{\mathbf{R}} \frac{P_{n}(y) P_{n-1}(y) w(y)}{y-t} d y,
\end{aligned}
$$

where we used (1.5). Substituting (3.7), (3.8), (3.9) and (3.10) into (3.5) and (3.6), the theorem is established.

Putting (3.1) and (3.2) into $\left(S_{1}\right)$, and comparing the constants and coefficients of $\frac{1}{z}$, respectively, we have

$$
2 \alpha_{n}=t+R_{n}(t)
$$

and

$$
r_{n+1}(t)+r_{n}(t)=\gamma+\left(t-\alpha_{n}\right) R_{n}(t) .
$$

Carrying out a similar calculation with $\left(S_{2}\right)$ and $\left(S_{2}^{\prime}\right)$, they give rise to another three identities:

$$
\begin{gathered}
1-r_{n}(t)+r_{n+1}(t)=2 \beta_{n+1}-2 \beta_{n} \\
\left(t-\alpha_{n}\right)\left(r_{n+1}(t)-r_{n}(t)\right)=\beta_{n+1} R_{n+1}(t)-\beta_{n} R_{n-1}(t)
\end{gathered}
$$

and

$$
\sum_{j=0}^{n-1} R_{j}(t)=2 \beta_{n}\left(R_{n-1}(t)+R_{n}(t)\right)-t r_{n}(t) .
$$


A telescopic sum of (3.13) gives

$$
n+r_{n}(t)=2 \beta_{n}
$$

Multiplying $R_{n}(t)$ on both sides of (3.14),

$$
\left(t-\alpha_{n}\right) R_{n}(t)\left(r_{n+1}(t)-r_{n}(t)\right)=\beta_{n+1} R_{n+1}(t) R_{n}(t)-\beta_{n} R_{n-1}(t) R_{n}(t),
$$

then eliminating $\left(t-\alpha_{n}\right) R_{n}(t)$ by (3.12), it yields

$\left(r_{n+1}^{2}(t)-r_{n}^{2}(t)-\gamma\right)\left(r_{n+1}(t)-r_{n}(t)\right)=\beta_{n+1} R_{n+1}(t) R_{n}(t)-\beta_{n} R_{n-1}(t) R_{n}(t)$.

A telescopic sum of above equation,

$$
r_{n}^{2}(t)-\gamma r_{n}(t)=\beta_{n} R_{n-1}(t) R_{n}(t) .
$$

Remark. (3.11), (3.12), (3.13), (3.14), (3.15), (3.16) and (3.17) are generated from $\left(S_{1}\right),\left(S_{2}\right)$ and $\left(S_{2}^{\prime}\right)$, which are significant for the next discussions.

\section{The $t$ dependance}

Mention again that the weight (1.2) depends on $t$, it means all of the quantities considered in this paper can be viewed as functions in $t$. In this section, we will investigate their dependance with respect to their parameter.

Taking a derivative with respect to $t$ in (1.4) when $i=j=n$,

$h_{n}(t, \gamma)=\int_{\mathbf{R}} P_{n}^{2}(z, t, \gamma) w(z . t, \gamma) d z=\int_{\mathbf{R}} P_{n}^{2}(z, t, \gamma) e^{-z^{2}+t z}|z-t|^{\gamma}(A+B \theta(z-t)) d z$,

we find

$$
\begin{aligned}
\frac{\partial h_{n}(t, \gamma)}{\partial t} & =\int_{\mathbf{R}} P_{n}^{2}(z, t, \gamma) w(z, t, \gamma) z d z+\int_{\mathbf{R}} \frac{\gamma P_{n}^{2}(z, t, \gamma) w(z, t, \gamma)}{t-z} d z \\
& =\alpha_{n} h_{n}-\gamma \int_{\mathbf{R}} \frac{P_{n}^{2}(z, t, \gamma) w(z, t, \gamma)}{z-t} d z \\
& =\alpha_{n} h_{n}-R_{n}(t) h_{n},
\end{aligned}
$$

where

$$
\frac{\partial\left(|z-t|^{\gamma}\right)}{\partial t}=\frac{\partial\left(|t-z|^{\gamma}\right)}{\partial t}=\left((t-z)^{\gamma}-(z-t)^{\gamma}\right) \delta(t-z)+\frac{\gamma|t-z|^{\gamma}}{t-z}
$$

and the definition of $R_{n}(t)$ are used. Straightforward,

$$
\frac{d \ln h_{n}(t)}{d t}=\frac{1}{h_{n}(t)} \frac{d h_{n}(t)}{d t}=\alpha_{n}-R_{n}(t)=\frac{t}{2}-\frac{R_{n}(t)}{2},
$$

where $\alpha_{n}$ is eliminated by (3.11).

It follows

$$
\begin{aligned}
\frac{d \ln \beta_{n}}{d t} & =\frac{1}{h_{n}(t)} \frac{d h_{n}(t)}{d t}-\frac{1}{h_{n-1}(t)} \frac{d h_{n-1}(t)}{d t} \\
& =\frac{1}{2}\left(R_{n-1}(t)-R_{n}(t)\right),
\end{aligned}
$$


where we used (1.8), and we can pose the derivative of $\beta_{n}$ by the difference between $R_{n-1}(t)$ and $R_{n}(t)$,

$$
\frac{d \beta_{n}}{d t}=\frac{\beta_{n}}{2}\left(R_{n-1}(t)-R_{n}(t)\right) .
$$

On the other hand, similarly steps, taking a derivative of

$$
\int_{\mathbf{R}} P_{n}(z, t, \gamma) P_{n-1}(z, t, \gamma) w(z, t, \gamma) d z=0
$$

with respect to $t$ and using the definition of $r_{n}(t)$, it produces

$$
\begin{aligned}
0= & \int_{\mathbf{R}} \frac{\partial P_{n}(z, t, \gamma)}{\partial t} P_{n-1}(z, t, \gamma) w(z, t, \gamma) d z+\int_{\mathbf{R}} P_{n}(z, t, \gamma) P_{n-1}(z, t, \gamma) w(z, t, \gamma) z d z \\
& +\int_{\mathbf{R}} P_{n}(z, t, \gamma) P_{n-1}(z, t, \gamma) e^{-z^{2}+t z} \frac{\partial\left(|z-t|^{\gamma}\right)}{\partial t}(A+B \theta(z-t)) d z \\
& +\int_{\mathbf{R}} P_{n}(z, t, \gamma) P_{n-1}(z, t, \gamma) e^{-z^{2}+t z}|z-t|^{\gamma} \frac{\partial(A+B \theta(z-t))}{\partial t} d z \\
= & h_{n-1} \frac{d \mathbf{p}(n, t)}{d t}+\beta_{n} h_{n-1}-\gamma \int_{\mathbf{R}} \frac{P_{n}(z) P_{n-1}(z) w(z)}{z-t} d z \\
= & h_{n-1} \frac{d \mathbf{p}(n, t)}{d t}+\beta_{n} h_{n-1}-r_{n}(t) h_{n-1},
\end{aligned}
$$

simplify,

$$
\frac{d \mathbf{p}(n, t)}{d t}=r_{n}(t)-\beta_{n} .
$$

Differential (1.7) and applying (4.3),

$$
\begin{aligned}
\alpha_{n}^{\prime}(t) & =r_{n}(t)-\beta_{n}-r_{n+1}(t)+\beta_{n+1} \\
& =\frac{1}{2}\left(r_{n}(t)-r_{n+1}(t)+1\right),
\end{aligned}
$$

since (3.16). We summarize in the following theorem.

Theorem 4.1. The recurrence coefficients $\alpha_{n}$ and $\beta_{n}$ are expressed in terms of $r_{n}(t), r_{n+1}(t), R_{n-1}(t)$ and $R_{n}(t)$ as follows:

$$
\beta_{n}^{\prime}(t)=\frac{\beta_{n}}{2}\left(R_{n-1}(t)-R_{n}(t)\right),
$$

and

$$
\alpha_{n}^{\prime}(t)=\frac{1}{2}\left(r_{n}(t)-r_{n+1}(t)+1\right) .
$$

Theorem 4.2. The auxiliary quantities $r_{n}(t)$ and $R_{n}(t)$ satisfy the coupled Riccati equations

$$
\begin{gathered}
r_{n}^{\prime}(t)=\frac{r_{n}^{2}(t)-\gamma r_{n}(t)}{R_{n}(t)}-\frac{\left(r_{n}(t)+n\right) R_{n}(t)}{2} \\
R_{n}^{\prime}(t)=2 r_{n}(t)-\frac{1}{2}\left(t-R_{n}(t)\right) R_{n}(t)-\gamma
\end{gathered}
$$


Proof. Taking a derivative of (3.16) with respect to $t$,

$$
2 \beta_{n}^{\prime}(t)=r_{n}^{\prime}(t)
$$

eliminating $\beta_{n}^{\prime}(t)$ by (4.4),

$$
r_{n}^{\prime}(t)=\beta_{n} R_{n-1}(t)-\beta_{n} R_{n}(t),
$$

then eliminating $\beta_{n} R_{n-1}(t)$ by (3.17), and eliminating another $\beta_{n}$ by (3.16), respectively, we arrive (4.6).

Combining (3.12) and (3.11), and eliminating $\alpha_{n}$,

$$
r_{n+1}(t)+r_{n}(t)=\gamma+\frac{1}{2}\left(t-R_{n}(t)\right) R_{n}(t) .
$$

Substituting (4.9) into (4.5), and eliminating $r_{n+1}(t)$,

$$
\alpha_{n}^{\prime}(t)=r_{n}(t)-\frac{1}{4}\left(t-R_{n}(t)\right) R_{n}(t)+\frac{1-\gamma}{2} .
$$

We note the derivative on both sides of (3.11),

$$
2 \alpha_{n}^{\prime}(t)=1+R_{n}^{\prime}(t)
$$

A simple computation with combining (4.10) and (4.11) and eliminating $\alpha_{n}^{\prime}(t)$, (4.7) is confirmed.

\section{$5 \quad \sigma-$ Form}

In this section, we introduce two auxiliary quantities $\sigma_{n}(t)$ and $\hat{\sigma}_{n}(t)$, and obtain their second order non-linear differential or difference equations.

Define a quantity allied to the Hankel determinant,

$$
\sigma_{n}(t):=\frac{d}{d t} \ln D_{n}(t)=\frac{d}{d t} \sum_{j=0}^{n-1} \ln h_{j}(t)=\sum_{j=0}^{n-1} \frac{d}{d t}\left(\ln h_{j}(t)\right) .
$$

Due to (4.2), (3.11) and (1.9),

$$
\sigma_{n}(t)=\frac{n t}{2}-\frac{1}{2} \sum_{j=0}^{n-1} R_{j}(t)=n t-\sum_{j=0}^{n-1} \alpha_{j}=\mathbf{p}(n, t, \gamma)+n t .
$$

Theorem 5.1. The quantity $\sigma_{n}(t)$ satisfies

$$
\left(\sigma_{n}^{\prime \prime}(t)\right)^{2}=\frac{1}{4}\left(t \sigma_{n}^{\prime}(t)-\sigma_{n}(t)\right)^{2}-4\left(\sigma_{n}^{\prime}(t)-\frac{n}{2}\right) \sigma_{n}^{\prime}(t)\left(\sigma_{n}^{\prime}(t)-\frac{n+\gamma}{2}\right),
$$

which is a particular Jimbo-Miwa-Okamoto $\sigma$-form of the Painlevé IV equation [15]. 
Proof. In view of (5.1),

$$
n t-2 \sigma_{n}(t)=\sum_{j=0}^{n-1} R_{j}(t) .
$$

Combining (3.15) and above equation,

$$
n t-2 \sigma_{n}(t)=2 \beta_{n} R_{n-1}(t)+2 \beta_{n} R_{n}(t)-t r_{n}(t),
$$

then eliminating $\beta_{n} R_{n-1}(t)$ and $\beta_{n}$ by (3.17) and (3.16), respectively,

$$
\left(n+r_{n}(t)\right) R_{n}(t)+\frac{2\left(r_{n}^{2}(t)-\gamma r_{n}(t)\right)}{R_{n}(t)}=t r_{n}(t)-2 \sigma_{n}(t)+n t .
$$

On account of (4.6),

$$
\frac{2\left(r_{n}^{2}(t)-\gamma r_{n}(t)\right)}{R_{n}(t)}-\left(n+r_{n}(t)\right) R_{n}(t)=2 r_{n}^{\prime}(t) .
$$

The sum of (5.4) and (5.5) gives

$$
\frac{4\left(r_{n}^{2}(t)-\gamma r_{n}(t)\right)}{R_{n}(t)}=t r_{n}(t)-2 \sigma_{n}(t)+n t+2 r_{n}^{\prime}(t) ;
$$

oppositely, (5.4) minus (5.5),

$$
2\left(n+r_{n}(t)\right) R_{n}(t)=t r_{n}(t)-2 \sigma_{n}(t)+n t-2 r_{n}^{\prime}(t) .
$$

Then the product of (5.6) and (5.7) is

$$
8 r_{n}(t)\left(r_{n}(t)+n\right)\left(r_{n}(t)-\gamma\right)=\left(t r_{n}(t)-2 \sigma_{n}(t)+n t\right)^{2}-4\left(r_{n}^{\prime}(t)\right)^{2} .
$$

Taking the derivative of (5.1) with respect to $t$ gives to

$$
\sigma_{n}^{\prime}(t)=\mathbf{p}^{\prime}(n, t, \gamma)+n,
$$

and eliminating $\beta_{n}$ between (3.16) and (4.3),

$$
\mathbf{p}^{\prime}(n, t, \gamma)=\frac{r_{n}(t)}{2}-\frac{n}{2} .
$$

Inserting (5.10) into (5.9), and eliminating $\mathbf{p}^{\prime}(n, t, \gamma)$,

$$
\sigma_{n}^{\prime}(t)=\frac{r_{n}(t)}{2}+\frac{n}{2}
$$

Some simplifications after substituting (5.11) into (5.8), we get (5.2) immediately.

Remark 4. $\sigma_{n}(t)$ satisfies the continuous $\sigma$-form of the Painleve $I V$, which theorem 5.1 is coincident with Tracy and Widom [20]. 
Proposition 5.2. We have

$$
\begin{aligned}
\sigma_{n}(t)= & \frac{d}{d t} \ln D_{n}(t) \\
= & -\frac{\left(R_{n}^{\prime}(t)\right)^{2}}{4 R_{n}(t)}+\frac{R_{n}^{3}(t)}{16}-\frac{t}{8} R_{n}^{2}(t)+\left(\frac{t^{2}}{16}-\frac{n}{2}-\frac{\gamma}{4}\right) R_{n}(t)+\frac{(2 n+\gamma) t}{4} \\
& +\frac{\gamma^{2}}{4 R_{n}(t)}
\end{aligned}
$$

Proof. For (3.15), eliminating $\beta_{n} R_{n-1}(t)$ and $\beta_{n}$ by (3.17) and (3.16),

$$
\sum_{j=0}^{n-1} R_{j}(t)=\frac{2\left(r_{n}^{2}(t)-\gamma r_{n}(t)\right)}{R_{n}(t)}+\left(n+r_{n}(t)\right) R_{n}(t)-t r_{n}(t) .
$$

Substituting (5.13) into (5.3),

$$
\sigma_{n}(t)=\frac{n t}{2}-\frac{r_{n}^{2}(t)-\gamma r_{n}(t)}{R_{n}(t)}-\frac{\left(n+r_{n}(t)\right) R_{n}(t)}{2}+\frac{t r_{n}(t)}{2} .
$$

Combining (4.7) and (5.14), and eliminating $r_{n}(t)$, the proof is completed.

Also define the other quantity

$$
\hat{\sigma}_{n}(t):=-\sum_{j=0}^{n-1} R_{j}(t) .
$$

Theorem 5.3. A second-order difference equation satisfied by $\hat{\sigma}_{n}(t)$,

$$
\begin{aligned}
& 2\left(n \hat{\sigma}_{n-1}+\hat{\sigma}_{n}-n \hat{\sigma}_{n+1}\right)^{2}-2 \gamma\left(n \hat{\sigma}_{n-1}+\hat{\sigma}_{n}-n \hat{\sigma}_{n+1}\right)\left(t-\hat{\sigma}_{n-1}+\hat{\sigma}_{n+1}\right) \\
= & \left(\hat{\sigma}_{n-1}-\hat{\sigma}_{n}\right)\left(\hat{\sigma}_{n}-\hat{\sigma}_{n+1}\right)\left(\hat{\sigma}_{n}+n t\right)\left(\hat{\sigma}_{n+1}-\hat{\sigma}_{n-1}+t\right),
\end{aligned}
$$

which is a discrete $\sigma$-form equation.

Proof. From (5.15),

$$
R_{n}(t)=\hat{\sigma}_{n}(t)-\hat{\sigma}_{n+1}(t),
$$

we can rewrite (3.15) with the aid of (3.16) and (5.17), i.e.

$$
\left(n+r_{n}(t)\right)\left(\hat{\sigma}_{n-1}(t)-\hat{\sigma}_{n+1}(t)\right)-t r_{n}(t)=-\hat{\sigma}_{n}(t),
$$

solving $r_{n}(t)$,

$$
r_{n}(t)=\frac{n\left(\hat{\sigma}_{n-1}(t)-\hat{\sigma}_{n+1}(t)\right)+\hat{\sigma}_{n}(t)}{t-\hat{\sigma}_{n-1}(t)+\hat{\sigma}_{n+1}(t)} .
$$

Similarly steps to recall (3.17) by using (3.16) and (5.17),

$$
r_{n}^{2}(t)-\gamma r_{n}(t)=\frac{1}{2}\left(n+r_{n}(t)\right)\left(\hat{\sigma}_{n-1}(t)-\hat{\sigma}_{n}(t)\right)\left(\hat{\sigma}_{n}(t)-\hat{\sigma}_{n+1}(t)\right) .
$$

Inserting (5.18) into (5.19) and simplify, it yields (5.16).

Remark. When $\gamma=0$, (5.16) is a discrete $\sigma$-form of Painlevé $\boldsymbol{I V}$ equation, which corresponds to Min and Chen [14]. 


\section{Painlevé IV and asymptotic behaviors}

Theorem 6.1. The quantity $R_{n}(t)$ satisfies the following second order differential equation:

$R_{n}^{\prime \prime}(t)=\frac{\left(R_{n}^{\prime}(t)\right)^{2}}{2 R_{n}(t)}+\frac{3}{8} R_{n}^{3}(t)-\frac{t}{2} R_{n}^{2}(t)+\frac{1}{8}\left(t^{2}-8 n-4-4 \gamma\right) R_{n}(t)-\frac{\gamma^{2}}{2 R_{n}(t)}$,

which is a particular Painlevé $I V$ equation. Moreover, let $\widetilde{R}_{n}:=\frac{1}{2} R_{n}(t)$ and $u:=-\frac{1}{4} t$, then

$$
\widetilde{R}_{n}^{\prime \prime}(u)=\frac{\left(\widetilde{R}_{n}^{\prime}(u)\right)^{2}}{2 \widetilde{R}_{n}(u)}+\frac{3}{2} \widetilde{R}_{n}^{3}(u)+4 u \widetilde{R}_{n}^{2}(u)+2\left(u^{2}-\theta_{1}\right) \widetilde{R}_{n}(u)+\frac{\theta_{2}}{\widetilde{R}_{n}(u)},
$$

which satisfies the Painlevé IV equation [11] with $\theta_{1}=\frac{2 n+1+\gamma}{4}, \theta_{2}=-\frac{\gamma^{2}}{8}$.

Proof. Solving for $r_{n}(t)$ from (4.7),

$$
r_{n}(t)=\frac{1}{2} R_{n}^{\prime}(t)+\frac{1}{4}\left(t-R_{n}(t)\right) R_{n}(t)+\frac{\gamma}{2},
$$

and substituting the solution into (4.6), simplify, we find (6.1).

Let $\widetilde{R}_{n}:=\frac{1}{2} R_{n}(t)$ and $u:=-\frac{1}{4} t$, it is to see that $\widetilde{R}_{n}(u)$ takes the form

$\widetilde{R}_{n}^{\prime \prime}(u)=\frac{\left(\widetilde{R}_{n}^{\prime}(u)\right)^{2}}{2 \widetilde{R}_{n}(u)}+\frac{3}{2} \widetilde{R}_{n}^{3}(u)+4 u \widetilde{R}_{n}^{2}(u)+2\left(u^{2}-\frac{n}{2}-\frac{1}{4}-\frac{\gamma}{4}\right) \widetilde{R}_{n}(u)-\frac{\gamma^{2}}{8 \widetilde{R}_{n}(u)}$.

Remark 5. A second-order differential equation satisfied by $r_{n}(t)$, which is related to the Chazy equation [12, 14], can also be obtained by solving for $R_{n}(t)$ from (4.6) and substituting the result of $R_{n}(t)$ into (4.7). We do not write it down due to its too complicated.

Disregard the derivative in (6.1) and get a quartic equation

$$
\frac{3}{8} R_{n}^{4}(t)-\frac{t}{2} R_{n}^{3}(t)+\frac{1}{8}\left(t^{2}-8 n-4-4 \gamma\right) R_{n}^{2}(t)-\frac{\gamma^{2}}{2}=0 .
$$

If $\gamma=0$ for (6.2),$X_{n}(t)$ satisfies a quadratic equation,

$$
3 X_{n}^{2}(t)-4 t X_{n}(t)+t^{2}-8 n-4=0,
$$

with the solutions

$$
X_{n}(t)=\frac{2 t \pm \sqrt{t^{2}+24 n+12}}{3} .
$$

Due to the definition of weight (1.2), we separate two parts to discuss the asymptotic behaviors of $R_{n}(t)$.

(i)If $B>0$, we choose

$$
X_{n}(t)=\frac{2 t+\sqrt{t^{2}+24 n+12}}{3},
$$


as $n \rightarrow \infty$,

$$
X_{n}(t)=\frac{2 \sqrt{6}}{3} n^{\frac{1}{2}}+\frac{2 t}{3}+\frac{12+t^{2}}{12 \sqrt{6}} n^{-\frac{1}{2}}-\frac{\left(12+t^{2}\right)^{2}}{1152 \sqrt{6}} n^{-\frac{3}{2}}+\mathcal{O}\left(n^{-\frac{5}{2}}\right) .
$$

Hence, we assume the expansion of $R_{n}(t)$, as $n \rightarrow \infty$,

$$
R_{n}(t)=\sum_{j=0}^{n-1} d_{j}(t) n^{\frac{1-j}{2}} .
$$

Substituting (6.3) into (6.1), we obtain

$$
\begin{aligned}
& d_{0}(t)=\frac{2 \sqrt{6}}{3}, \quad d_{1}(t)=\frac{2 t}{3}, \quad d_{2}(t)=\frac{\sqrt{6}\left(t^{2}+12 \gamma+12\right)}{72}, \quad d_{3}(t)=0, \\
& d_{4}(t)=\frac{\sqrt{6}\left(288 \gamma^{2}-24 t^{2} \gamma-288 \gamma-t^{4}-24 t^{2}-240\right)}{6912}, \quad d_{5}(t)=\frac{\left(2-9 \gamma^{2}\right) t}{72},
\end{aligned}
$$

which means

$$
\begin{aligned}
R_{n}(t)= & \frac{2 \sqrt{6}}{3} n^{\frac{1}{2}}+\frac{2 t}{3}+\frac{\sqrt{6}\left(t^{2}+12 \gamma+12\right)}{72} n^{-\frac{1}{2}} \\
& +\frac{\sqrt{6}\left(288 \gamma^{2}-24 t^{2} \gamma-288 \gamma-t^{4}-24 t^{2}-240\right)}{6912} n^{-\frac{3}{2}}+\frac{\left(2-9 \gamma^{2}\right) t}{72} n^{-2} \\
& +\mathcal{O}\left(n^{-\frac{5}{2}}\right) .
\end{aligned}
$$

(ii)If $B<0$, we choose

$$
X_{n}(t)=\frac{2 t-\sqrt{t^{2}+24 n+12}}{3},
$$

as $n \rightarrow \infty$,

$$
X_{n}(t)=-\frac{2 \sqrt{6}}{3} n^{\frac{1}{2}}+\frac{2 t}{3}-\frac{12+t^{2}}{12 \sqrt{6}} n^{-\frac{1}{2}}+\frac{\left(12+t^{2}\right)^{2}}{1152 \sqrt{6}} n^{-\frac{3}{2}}+\mathcal{O}\left(n^{-\frac{5}{2}}\right) .
$$

Similarly, we assume the expansion of $R_{n}(t)$, as $n \rightarrow \infty$,

$$
R_{n}(t)=\sum_{j=0}^{n-1} \widetilde{d}_{j}(t) n^{\frac{1-j}{2}} .
$$

Substituting (6.4) into (6.1), we obtain

$$
\begin{aligned}
& \widetilde{d}_{0}(t)=-\frac{2 \sqrt{6}}{3}, \quad \widetilde{d}_{1}(t)=\frac{2 t}{3}, \quad \widetilde{d}_{2}(t)=-\frac{\sqrt{6}\left(t^{2}+12 \gamma+12\right)}{72}, \quad \widetilde{d}_{3}(t)=0, \\
& \widetilde{d}_{4}(t)=-\frac{\sqrt{6}\left(288 \gamma^{2}-24 t^{2} \gamma-288 \gamma-t^{4}-24 t^{2}-240\right)}{6912}, \quad \widetilde{d}_{5}(t)=\frac{\left(2-9 \gamma^{2}\right) t}{72},
\end{aligned}
$$

which means

$$
\begin{aligned}
R_{n}(t)= & -\frac{2 \sqrt{6}}{3} n^{\frac{1}{2}}+\frac{2 t}{3}-\frac{\sqrt{6}\left(t^{2}+12 \gamma+12\right)}{72} n^{-\frac{1}{2}} \\
& -\frac{\sqrt{6}\left(288 \gamma^{2}-24 t^{2} \gamma-288 \gamma-t^{4}-24 t^{2}-240\right)}{6912} n^{-\frac{3}{2}}+\frac{\left(2-9 \gamma^{2}\right) t}{72} n^{-2} \\
& +\mathcal{O}\left(n^{-\frac{5}{2}}\right) .
\end{aligned}
$$

Straightforward, we summarize the following item. 
Theorem 6.2. As $n \rightarrow \infty$, the quantity $R_{n}(t)$ has the asymptotic expansions:

- if $B>0$

$$
\begin{aligned}
R_{n}(t)= & \frac{2 \sqrt{6}}{3} n^{\frac{1}{2}}+\frac{2 t}{3}+\frac{\sqrt{6}\left(t^{2}+12 \gamma+12\right)}{72} n^{-\frac{1}{2}} \\
& +\frac{\sqrt{6}\left(288 \gamma^{2}-24 t^{2} \gamma-288 \gamma-t^{4}-24 t^{2}-240\right)}{6912} n^{-\frac{3}{2}}+\frac{\left(2-9 \gamma^{2}\right) t}{72} n^{-2} \\
& +\mathcal{O}\left(n^{-\frac{5}{2}}\right) ;
\end{aligned}
$$

- if $B<0$

$$
\begin{aligned}
R_{n}(t)= & -\frac{2 \sqrt{6}}{3} n^{\frac{1}{2}}+\frac{2 t}{3}-\frac{\sqrt{6}\left(t^{2}+12 \gamma+12\right)}{72} n^{-\frac{1}{2}} \\
& -\frac{\sqrt{6}\left(288 \gamma^{2}-24 t^{2} \gamma-288 \gamma-t^{4}-24 t^{2}-240\right)}{6912} n^{-\frac{3}{2}}+\frac{\left(2-9 \gamma^{2}\right) t}{72} n^{-2} \\
& +\mathcal{O}\left(n^{-\frac{5}{2}}\right) .
\end{aligned}
$$

By direct computations, we arrive at the following expansion formula for the scaled Hankel determinant

Theorem 6.3. For fixed $s>0, \gamma>-1$, we obtain

- if $B>0$, as $n \rightarrow \infty$,

$$
\begin{aligned}
\ln \frac{D_{n}(s)}{D_{n}(0)}= & -\frac{2 \sqrt{6}}{9} s n^{\frac{3}{2}}+\frac{s^{2} n}{12}-\frac{\sqrt{6}\left(s^{3}+36 \gamma s\right) n^{\frac{1}{2}}}{216}+\frac{s^{4}}{864}+\frac{s^{2} \gamma}{24} \\
& +\mathcal{O}\left(n^{-\frac{1}{2}}\right)
\end{aligned}
$$

- if $B<0$, as $n \rightarrow \infty$,

$$
\begin{aligned}
\ln \frac{D_{n}(s)}{D_{n}(0)}= & \frac{2 \sqrt{6}}{9} s n^{\frac{3}{2}}+\frac{s^{2} n}{12}+\frac{\sqrt{6}\left(s^{3}+36 \gamma s\right) n^{\frac{1}{2}}}{216}+\frac{s^{4}}{864}+\frac{s^{2} \gamma}{24} \\
& +\mathcal{O}\left(n^{-\frac{1}{2}}\right)
\end{aligned}
$$

Proof. Putting (6.5) into (5.12), the logarithmic derivative of the Hankel determinant $D_{n}(t)$ for $B>0$ becomes,

$$
\begin{aligned}
\frac{d}{d t} \ln D_{n}(t)= & -\frac{2 \sqrt{6}}{9} n^{\frac{3}{2}}+\frac{n t}{6}-\frac{\sqrt{6}\left(t^{2}+12 \gamma\right) n^{\frac{1}{2}}}{72}+\frac{t^{3}}{216}+\frac{t \gamma}{12} \\
& +\mathcal{O}\left(n^{-\frac{1}{2}}\right) .
\end{aligned}
$$

Then, integration on both sides of equation (6.9) from 0 to $s$,

$$
\int_{0}^{s} \frac{d}{d t} \ln D_{n}(t) d t=\ln D_{n}(s)-\ln D_{n}(0),
$$

we obtain (6.7). The proof is similar for $B<0$. 
Once the asymptotic behaviors for $R_{n}(t)$ is obtained, we also consider the large $n$ behavior of the monic orthogonal polynomials $P_{n}(z)$.

Theorem 6.4. As $n \rightarrow \infty$ and $t \rightarrow \infty, P_{n}(z)$ satisfies the second order differential-difference equation,

$P_{n}^{\prime \prime}(z)+\left(\frac{3}{z}-2 z+t\right) P_{n}^{\prime}(z)+\left(\left(\frac{2 t^{2}}{3}+\frac{\gamma}{12}\right) \frac{n^{2} \gamma}{z^{6}}+\frac{2 n^{2} \gamma t}{3 z^{5}}+\frac{2 n^{2} \gamma}{3 z^{4}}\right) P_{n}(z)=0$.

Proof. Substituting (3.1) and (3.2) into (2.17) and (2.18), $Q_{n}(z)$ and $T_{n}(z)$ can be expressed in terms of $r_{n}(t), R_{n}(t)$ and $\beta_{n}$. It should be pointed out that relationship among $R_{n}(t), r_{n}(t), \alpha_{n}$ and $\beta_{n}$ are shown by (3.11), (3.16) and (4.7). The coefficients of (2.16) are given in terms of $R_{n}(t)$. Sending $n \rightarrow \infty, t \rightarrow \infty$, and combining the asymptotic value (6.5) or (6.6), we obtain (6.10).

Remark 6. When $\gamma=0$, (6.10) satisfies the bi-confluent Heun equation. More details of the Heun equation, see [5, 10, 16, 18, 19, 21]].

\section{Acknowledgements}

D. Wang, M. Zhu and Y. Chen would like to give thanks the Science and Technology Development Fund of the Macau SAR for providing FDCT 023/ 2017/A1. They would also like to thank the University of Macau for MYRG 2018-00125 FST.

\section{Availability of data}

The data that support the findings of this study are available from the corresponding author upon reasonable request.

\section{References}

[1] Y. Chen and G. Pruessner, Orthogonal polynomials with discontinuous weights, J. Phys. A: Math. Gen., 38 (2005) 191-198.

[2] Y. Chen and M. E. H. Ismail, Ladder operators and differential equations for orthogonal polynomials, J. Phys. A: Math. Gen., 30 (1997) 78187829.

[3] Y. Chen and M. E. H. Ismail, Jacobi polynomials from compatibility conditions, Proc. Amer. Math. Soc., 133 (2015) 465-472.

[4] Y. Chen and M. V. Feigin, Painlevé IV and degenerate Gaussian unitary ensembles, J. Phys. A: Math. Gen., 39 (2006) 12381-12393.

[5] Y. Chen, G. Filipuk and L. Zhan, Orthogonal polynomials, asymptotics and Heun equation, J. Math. Phys., 60 (2019) 113501. 
[6] Y. Chen, N. S. Haq and M. R. Mckay, Random matrix models, doubletime Painlevé equations, and wireless relaying, J. Phys. A.: Math. Gen., 54 (2013) 063506.

[7] Y. Chen and N. Lawrence, On the linear statistics of Hermitian random matrices, J. Phys. A.: Math. Gen., 31 (1998) 1141-1152.

[8] Y. Chen and L. Zhang, Painlevé VI and the unitary Jacobi ensembles, Stud. Appl. Math., 125 (2010) 91-112.

[9] G. Filipuk, W. Van Assche and L. Zhang, The recurrence coefficients of semi-classical Laguerre polynomials and the fourth Painlevé equation, J. Phys. A: Math. Theor., 45 (2012) 205201.

[10] K. Heun, Zur theorie der Riemann'schen functionen zweiter ordnung mit vier verzweigungspunkten, (German) Math. Ann., 33 (1888) no. 2, 161-179.

[11] V. I. Gromak, I. Laine and S. Shimomura, Painlevé Differential Equations in the Complex Plane, Berlin, New York: Walter de Gruyter, 2002.

[12] S. Lyu and Y. Chen, The largest eigenvalue distribution of the Laguerre unitary ensemble, Acta Math. Sci., 37B (2017) 439-462.

[13] M. L. Mehta, Random Matrices, 3rd edn. (Elsevier, Singapore, 2006).

[14] C. Min and Y. Chen, Painlevé transcendents and the Hankel determinants generated by a discontinuous Gaussian weight, Math. Methods in Appl. Sci., 42 (2019) 301-321.

[15] M. Jimbo and T. Miwa, Monodromy perserving deformation of linear ordinary differential equations with rational coefficients. II, Physica D: Nonlinear Phenom, 2 (1981) 407-448.

[16] A. Ronveaux, Heun's Differential Equations, Oxford Science Publications. The Clarendon Press, Oxford University Press, New York, 1995.

[17] G. Szegö, Orthogonal Polynomials, American Mathematical society Colloquium Publication, vol. 23, (American Mathematical Society, New York, 1939)

[18] S. Y. Slavyanov and W. Lay, special Functions. A Unified Theory Based on Singularities, Oxford University Press, Oxford, 2000.

[19] B. D. Sleeman and V. B. Kuznetsov, Heun functions, NIST handbook of mathematical functions, 709-721, U.S. Dept. Commerce, Washington, D.C., 2010.

[20] C. A. Tracy and H. Widom, Fredholm determinants, differential equations and matrix models, Commun. Math. Phys., 163 (1994) 33-72.

[21] D. Wang, M. Zhu and Y. Chen, Orthogonal polynomials, bi-confluent Heun equations and semi-classical weights, J. Differ. Equ. Appl., 26(7) (2020) 1000-1012.

[22] D. Wang, M. Zhu and Y. Chen, On Semi-classical Orthogonal Polynomials Associated with a Freud-type Weight, Math. Meth. Appl. Sci., 43 (2020), 5295-5313. 
[23] L. Zhan, G. Blower, Y. Chen and M. Zhu, Center ofmass distribution of the Jacobi unitary ensembles: Painlevé $V$, asymptotic expansions, J. Math. Phys., 59(10) (2018) 103301.

[24] M. Zhu and Y. Chen, On properties of a deformed Freud weight, Random Matrices Theory Appl., 8 (2019) 1950004. 